\title{
PRIMER ENCUENTRO DE SEMILLEROS DE INVESTIGACIÓN DE LAS FACULTADES DE MEDICINA DE COLOMBIA
}

\section{Guillermo Sánchez V.MD*}

El 25, 26 y 27 de julio de 2012 se llevó a cabo el Primer Encuentro Nacional de Semilleros de Investigación de Facultades de Medicina en las instalaciones del centro de eventos de la biblioteca Luis Ángel Arango de Bogotá DC, con la participación de 39 facultades del país. Se presentaron 219 trabajos y asistieron cerca de 1.200 asistentes entre estudiantes y docentes en los tres días del encuentro.

Contamos con la participación de conferencistas nacionales e internacionales como la doctora Martha Lucía Ospina Viceministra de Salud y los doctores Manuel Elkin Patarroyo director del Instituto Nacional de Inmunología de Colombia, Eduardo Posada presidente de la Asociación Colombiana para el Avance de la Ciencia, Arturo Juárez y José Moreno investigadores del Instituto Nacional de Salud Pública de México y la investigadora Ingris Peláez del Hospital General de México. Además concurrieron docentes líderes de grupos de semilleros de las universidades Industrial de Santander, Antioquia y Unisangil, los cuales compartieron sus experiencias en la administración y orientación de la investigación en el pregrado.

Este escenario constituyó el primer espacio nacional que convocó a todas las facultades de medicina para la participación de sus estudiantes como investigadores, donde compartieron y socializaron sus productos con sus pares de otras instituciones.

Para la Fundación Universitaria de Ciencias de la Salud y los asistentes al encuentro, este espacio logró reconocer e incentivar la generación del nuevo conocimiento por parte de los estudiantes de pregrado. Es una estrategia generadora de cultura, que ha permitido el crecimiento institucional en todo lo que compete al desarrollo de la investigación en salud como un ele-

* Director División de Investigaciones Fundación Universitaria de Ciencias de la Salud.Bogotá DC, Colombia.division.investigaciones@fucsalud.edu.co

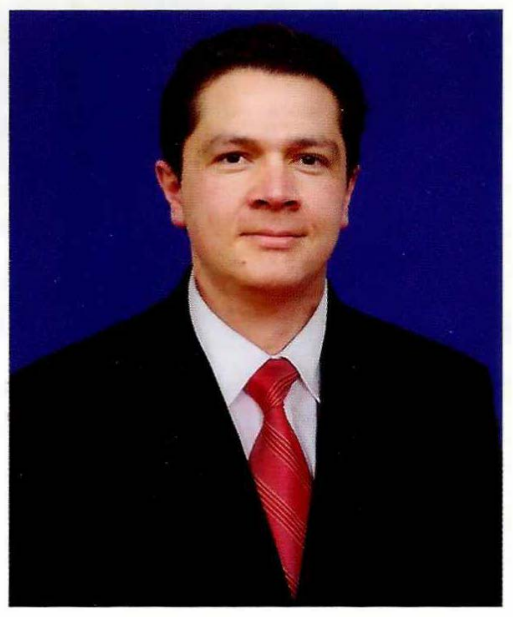

mento indispensable en la formación de profesionales integrales. En esta forma, la FUCS logra posicionarse como una de las instituciones pioneras en el desarrollo de competencias y habilidades para la investigación en Colombia.

Se otorgaron tres reconocimientos a las mejores ponencias orales y afiches (póster), con premios que apoyan la formación académica. Los resultados de este encuentro mostraron el interés masivo de los estudiantes de medicina por la participación en este tipo de escenarios, por lo cual la FUCS ya se encuentra estructurando el próximo evento que se realizaría para las mismas fechas del próximo año y en forma permanente.

La participación de los estudiantes de la FUCS como asistentes y ponentes es motivo de orgullo, convirtiéndose en un claro compromiso con el alma mater, apoyando y participando en las actividades desarrolladas por los integrantes de todos nuestros semilleros de investigación.

La División de Investigaciones agradece a todas las directivas, personal administrativo, docentes y estudiantes, el apoyo en este evento y los invita a participar el próximo año. 


\section{TRABAJOS CATEGORÍA CARTEL (póster)}

1er. Premio: Universidad del Rosario. Izquierdo Y.E, Fonseca E.V, Calvo E, Amaya J, Mantilla Rubén D, Anaya J.M, Rojas A. Factores asociados al progreso radiológico de la artritis reumatoide en pacientes latinoamericanos.

$2^{\circ}$. Premio: Universidad de Los Andes. Lemus N, Triana C, Gutiérrez L, Pedraza C, Grijalba C, Sarmiento O.L, Ramírez A, Pinzón P, Forero Y. Deficiencia de hierro durante la gestación en las mujeres colombianas; un problema de salud pública.

3er. Premio: Universidad Militar, Universidad de La Salle. Rodríguez A, Pachón D, Méndez I.A, Cabrera L.A. Leptospirosis en población de riesgo, Bogotá, Colombia.

Corporación Universitaria Rafael Núñez. Pinto D.A, Celis A.J, Alvis A, Liñan F. Asociación entre el estado nutricional y las enfermedades más frecuente en niños de 0 a 5 años de edad que asisten al centro de atención primaria La Esperanza en la ciudad de Cartagena.

Corporación Universitaria Remington. Rave L.B, Daza V.J, Arango J.C, Conocimientos básicos de los médicos generales acerca de las implicaciones de la enfermedad periodontal en embarazo.

Fundación Universitaria de Ciencias de la Salud. Boada L, Pedraza O, Sánchez E, Saavedra S, Sánchez A, Montalvo C, Plata S.J, Arévalo I. Previniendo y protegiendo la cognición del adulto mayor "PPCAM": cartilla guía para el paciente con deterioro cognitivo Leve (DCL).

Fundación Universitaria de Ciencias de la Salud. Castillejo J.E, Suárez M.A, Gutiérrez J.A. Tierradentro M.K. Sepsis neonatal por streptococcus pneumoniae: reporte de caso.

Fundación Universitaria de Ciencias de la Salud. Cortés SG. Análisis del comportamiento del examen de calidad de la educación superior (ECAES), frente al desempeño académico de los estudiantes de instrumentación quirúrgica en una IES. Bogotá, Colombia.

Fundación Universitaria de Ciencias de la Salud. Mora S.L, Peña M.S, Ocampo A. Caracterización biopsicosocial de la población de adolescentes embarazadas afiliadas a Salud Total EPS-S en la ciudad de Bogotá primer semestre 2011

Fundación Universitaria de Ciencias de la Salud. Niño C.G. Estudio de costo-efectividad de buprenorfina transdérmico versus oxicodona oral y fentanilo transdérmico en el manejo del dolor crónico no oncológico en el Hospital de San José de Bogotá D.C. enero-diciembre 2010.

Fundación Universitaria de Ciencias de la Salud. Picón E.S, Salgado H, Saavedra M.A. Perfil epidemiológico del servicio de urgencias según motivos de consulta del Hospital de San José centro en el período septiembre 2011 a febrero de 2012

Fundación Universitaria de Ciencias de la Salud. Preciado J.A, Jiménez H.J, Quintero J, Laza C. Experiencias y sentimientos vividos durante una gestación de alto riesgo: un estudio documental 2005-2011.

Fundación Universitaria de Ciencias de la Salud. Saavedra N, Velandia S, Murcia M.C, Suarez M.A, Durán E, Uribe M.C, García D. Frecuencia de alteraciones clínicas y paraclínicas en recién nacidos con $\mathrm{pH}$ de gases arteriales de cordón umbilical menor o igual a 7,18 en el Hospital de San José de diciembre de 2009 a marzo de 2012.

Fundación Universitaria de Ciencias de la Salud. Sánchez A, Pedraza O.L, Boada L, Sánchez E, Saavedra S, Montalvo C, Plata S.J, Arévalo I. Previniendo y protegiendo la cognición del adulto Mayor "PPCAM": Cartilla Guía para proteger las funciones intelectuales del adulto mayor sano.

Fundación Universitaria San Martín- Puerto Colombia. Tapia A, Viloria E, Camargo L, Alcocer A. Hallazgos morfológicos de ateroma en cadáveres en el Instituto Colombiano de Medicina Legal.
Fundación Universitaria San Martín.Puerto Colombia. Guerrero A, De la Rosa B, Mejia G, Vargas R. Prevalencia de dispareunia y factores de riesgos asociados en mujeres de una localidad del caribe colombiano.

Fundación Universitaria San Martín-Cali. Cifuentes A.F, Zapata H.de J. Incidencia de la mortalidad postneonatal y factores relacionados, Valle de Cauca año 2007.

Fundación Universitaria San Martín-Cali. Chávez J.B, Cubides A. Conocimientos de los cuidadores de menores de cinco años, respecto a la enfermedad respiratoria agua Santiago de Cali 2009.

Fundación Universitaria San Martín-Cali. Espinosa K, Hernández M. Factores asociados al consumo de sustancias psicoactivas en una institución educativa de Jamundí Valle. Año 2012.

Fundación Universitaria San Martín-Cali. Moreno F, Suárez R, Montoya C, Pineda M, Torres J, Hernández M. Factores sociodemográficos asociados a la violencia sexual en la ciudad de Cali, año 2009.

Fundación Universitaria San Martín-Pasto. Martínez D.A, Porras C, Gaviria A, Arboleda A.F, Patrones de riesgo y perjudiciales para el consumo de alcohol en estudiantes de medicina.

Fundación Universitaria San Martín-Pasto. Valenzuela A.M. Determinantes de adherencia al tratamiento antihipertensivo de adultos mayores a 35 años de edad.

Grupo de Investigación en Salud, Eduación y Profesionalismo S.E.P. - Línea de Atención Primaria en Salud. Jr. O’Brien A.T, Trujillo E.M, Olaya C. Metaanálisis de las intervenciones organizacionales en pacientes con hipertensión arterial que emplean los principios de las ciencias de la complejidad.

U.D.C.A. Espinosa R, Pardo H.A, Rojas S. Estandarización de la amplificación de los genes XRCC1 y XRCC3 por PCR en el laboratorio de biología molecular de la U.D.C.A

Unidad Central del Valle del Cauca. Muriel P, Gulloso L, Escobar S.E, Girón K.D, Maturana K. Y, Orobio C.P. Severidad de la violencia de pareja notificada en el municipio de Guadalajara de Buga - Valle.

Universidad Autónoma de Bucaramanga. Salazar D.S, Vásquez L.E, Castro M.F, Pérez C.E, Cadena L.del P. Determinantes del inicio del consumo de tabaco en estudiantes universitarios.

Universidad Cooperativa de Colombia. Arango D, Jiménez A, Cardona J.A. Prevalencia de coinfección VIH/TB 1997-2012: Revisión sistemática.

Universidad Cooperativa de Colombia. Higuita E, Cardona J.A, Conocimientos actitudes y prácticas sobre hepatitis B en estudiantes de una Facultad de Medicina de Medellín, 2011.

Universidad Cooperativa de Colombia. Monsalve K, Marriaga A.D, Osorio A.C, Chávez F.D, García S.P, Viera W.E, Cardona J.A. Prevalencia de insomnio y somnolencia en estudiante de una facultad de medicina de Medellín y su asociación con algunas condiciones sociodemográficas y académicas, 2012.

Universidad Cooperativa de Colombia. Salinas J.J, Martínez C.M, Murillo O.J. Estudio de prevalencia de los factores de riesgo, asociados al aumento de la tensión arterial en la comunidad académica de la Universidad Cooperativa de Colombia sede Medellín.

Universidad Cooperativa de Colombia-Pasto. Vanegas L.A, Vallejo V.H, Santacruz C, Valencia K.A. Prevalencia de anemia en adolescentes de sexo femenino entre los 10 a 19 años de edad del Centro Educativo de Integración Popular del barrio Chapal de la ciudad de Pasto - 2012.

Universidad de Boyacá. Pérez I.L, Becerra K, Bustamante M, Millán C, Riveros E. El pretratamiento con alopurinol disminuye la translocación bacteriana y los atenúa los cambios morfológicos de la mucosa intestinal en un modelo Murino de isquemia-reperfusión intestinal. 
Universidad de Boyacá. Zambrano P, Amado P, Riveros E. Adherencia a las guías de higiene de manos en cuidado intensivo en la Clínica de Los Andes de Tunja.

Universidad de Caldas. Duque J.E, Castaño L.F, González A. Variaciones morfológicas del músculo plastima en la población caldense (estudio in vivo sin disección)

Universidad de Cartagena. Palacio B, Rojas J. Prevalencia de período de eclampsia en Colombia. Informe preliminar.

Universidad de La Sabana. Montaño G.S, Gómez M, Páez V, Emmanuelli J, Domínguez M.T, Celis LG, Obregón M.C, Ayala J, Giratá V, Almonacid C.C. Caracterización antropométrica y de composición corporal de una población de estudiantes universitarios para el desarrollo de una cultura nutricional basada en el desarrollo de hábitos saludables.

Universidad de Los Andes, Fundación Santa Fe, Centers for Disease Control and Prevention, USA. Universidad Nacional de Colombia, Universidad de Hong Kong China, Universidad de Córdoba, Secretaria de Salud. Acosta JC, Ramírez A, Diaz MP, Sarmiento O, Duperly J, Lobelo F. Rojas N, W. Wong T, Arango C.M, Maldonado A, Aristizabal G. Acondicionamiento cardiorespiratorio en niños y contaminación del aire por material particulado en Bogotá.

Universidad de Los Andes, Pontificia Universidad Católica de Valparaiso, Pontificia Universidad Javeriana. Finkelsztein E.J, Guzmán F, Cuellar A, Puerta C, González J. Incremento de la inmunogenicidad de un péptido sintético derivado de la proteína KMP-11 de Trypanosoma cruzzi.

Universidad de Los Andes. Cárdenas L.L, Cano N, Gutiérrez C, Donado C, Duperly J. Consumo de sustancias con alto riesgo cardiovascular en deportistas universitarios de Bogotá.

Universidad de Los Andes. Durán G.A, Vargas J.C, González J.M. Efectos de la infección y antígenos solubles de Trypanosoma cruzien una línea celular de astrocitos humanos.

Universidad de Nariño. Delgado D, Arteaga J, Guancha A.L. Prevalencia de exposición a pesticidas en madres de niños diagnosticados con leucemia manejados en el Hospital Los Angeles de Pasto en tratamiento durante el año 2012 .

Universidad de Pamplona. Sarrazola D.M, Portilla H.C, Cabezas Z, Duran A. Concordancia inter-observador en auscultación pulmonar mediante simuladores en estudiantes de medicina de Ia Universidad de Pamplona.

Universidad de Santander (UDES). Molina I, Villamizar M, Celedón J, Bermúdez M. Efecto cataléptico de inhibidores de NO muestra expresión diferencial de proteína FOS.

Universidad del Magdalena. Canova C.J, Buendía M, Acosta D, De la Hoz M, Lapeira P, González T, Ramos E. Diagnóstico situacional de la salud mental de los habitantes del barrio Las Malvinas, Santa Marta. (I Etapa 2012-I).

Universidad del Magdalena. Cantillo K, Rico K, Alvarez L, Salazar A. Calidad y acceso al agua potable en Santa Marta como indicador de inequidad en salud.

Universidad del Mgdalena. Fernández M.F, Ledesma R.L, Alvarez L. Salazar A. Comportamiento de la transmisión del dengue y su relación con los períodos secos y lluviosos en Colombia, 2008-2010.

Universidad del Rosario. Bermúdez P.A, Del Riesgo L, Salamanca A.L, Monterey P, Vélez J.L. Factores ginecoobstétricos y condiciones periparto asociadas a Hipoxia Perinatal.

Universidad del Rosario. Camargo D.A, Barrera J, Mancera S. Discapacidad y sexualidad: "una mirada desde los cuidadores y personas con discapacidad localidades Chapinero - Barrios Unidos" Bogotá - Colombia.

Universidad del Rosario. Salazar LC, Speck C, De la Cuesta J, Quintero P, Parra R, Guarín M, Zapata E, Velandia L, Carrillo D, Torralvo G, Amaya
J, Herrena C, San Vicente Z, Anaya J.M, Montoya G, Rojas A. Factores de riesgo asociados a enfermedad cardiovascular: Experiencia a partir de la descripción de una muestra de individuos colombianos.

Universidad del Rosario: Sarmiento J.C, Amaya J, Herrera C, Espinosa J.S, Mantilla R.D, Anaya J.M, Villaraga A. Enfermedad cardiovascular en artritis reumatoide en América Latina: estudio de corte transversal de una nueva cohorte de pacientes colombianos y revisión sistemática de la literatura.

Universidad del Sinú. Gandía J.A, Benjumea Y, Mangones L.M, Villacob K.P, Sánchez L, Mosquera E. Prevalencia de Staphylococcus aureus meticilino resistente en estudiantes de medicina de la Universidad del Sinú.

Universidad del Tolima. Muñoz R, Cabezas D.C, Echeverri N, Murcia P.A, Hernández N, Méndez G. Análisis etnográfico de historias de vida con antecedentes delictivos.

Universidad del Valle. Gómez G.S, Morales F.J, Bonilla F.J, Fandiño C.A, Santaella J, Gutiérrez M.I, Riesgo asumido por los usuarios viales vulnerables: infracciones de las normas de tránsito en una ciudad capital del suroccidente colombiano 2009.

Universidad del Valle. Mendoza D.M, Marín D.J, Rojas Ch.A, Torres J, Echandia C.A. Factores de riesgo asociados al desarrollo de asfixia perinatal.

Universidad Industrial de Santander (UIS). Rincón L, Vargas M.C, Vera A, Meléndez H, Rueda O.L. Variables pronósticas de complicaciones de eventos cardiovasculares en cirugía no cardíaca.

Universidad Industrial de Santander (UIS). Sierra J.A, Puerto J.A, García E. Uso de inmunomarcadores para la determinación del pronóstico y respuesta al tratamiento en un caso de linfoma B difuso extranodal.

Universidad Industrial de Santander. Bello A, Bolivar A, Roberto S, Rueda O. Presencia de Neurofobia en estudiantes de medicina de una universidad colombiana.

Universidad Libre Seccional Barranquilla. Jiménez S.L, Escobar L, Fontalvo M, Mendoza E. Evaluación del estado nutricional y sobrecrecimiento bacteriano intestinal en una muestra niños entre 5 y 10 años del corregimiento de Juan Mina, Atlántico.

Universidad Militar Nueva Granada: Rodríguez J. Prieto S, Correa C, Bernal P, Tapia D, Alvarez L, Mora J, Vitery S, Salamanca D. Diagnóstico fractal de disfunción cardíaca severa, dinámica fractal de la ramificación coronaria izquierda.

Universidad Militar Nueva Granada: Rodríguez J, Vitery S, Puerta G, Muñoz D, Rojas I, Pinilla L, Mora J, Salamanca D, Perdomo N. Dinámica probabilista temporal de la epidemia de dengue en Colombia.

Universidad Militar Nueva Granada. Rodríguez J. Prieto S, Correa C, Pérez C, Mora J, Bravo J, Soracipa Y, Alvarez L. Predicción del recuento de linfocitos CD4 en pacientes con VIH a partir del hemograma.

Universidad Militar Nueva Granada. Rodríguez J, Alvarez L, Tapia D, López F, Cardona D.M, Mora J, Acuña C, Torres V, Pineda D, Rojas N. Evaluación de la dinámica cardíaca de pacientes con arritmia con base en la teoría de la probabilidad.

Universidad Nacional de Colombia, Universidad de Los Andes. Pabón A, Quintana G, Medina DL, Bacaret W, Quintana G. Experiencia en el uso de DMARDs no biológicos en un grupo de pacientes colombianos con artritis reumatoide temprana y establecida.

Universidad Simón Bolivar-Barranquilla. Maldonado J, Barraza D, Carriazo L, Castellar C, López C, Cano L.C. Factores biopsicosociales asociados a embarazos en adolescentes, Hospital Materno Infantil de Soledad 2000, enero-junio de 2011. 


\section{TRABAJOS CATEGORÍA PRESENTACIÓN ORAL}

1er. Premio: Universidad Tecnológica de Pereira (UTP). Ochoa S.A, Moreno P.A. Trastornos de la conducta alimentaria en adolescentes de colegios públicos de Pereira, 2011.

$2^{\circ}$. Premio: Universidad del Rosario. Calixto O.J, Amaya J, Quintero O.L, Amador M.J, Herrera C, Cárdenas J, Giraldo J, Barragán C, Caro J, De San Vicente Z, Cruz P, Mantilla R.D, Rojas A, Anaya J,M. Influencia del estrato socieconómico en enfermedades autoinmunes.

3er. Premio: Universidad Industrial de Santander. Fragozo M.C, Mendoza T, Vargas L.M, Zambrano N, Mendoza A, Rueda O.L. Variabilidad de la frecuencia cardíaca (VFC) como variable pronóstica de morbi-mortalidad en la fase aguda (intrahospitalaria) del infarto del miocardio.

Corporación Universitaria Remington. Cardona S, Porras C.P, Choles E, Zuluaga R, Arango J.C. Conocimiento de las madres de niños menores de 5 años acerca del uso de acetaminofén en el manejo de la fiebre y el riesgo de su toxicidad en el servicio de AIEPI de una unidad hospitalaria de segundo nivel.

Fundación Universitaria de Ciencias de la Salud. Arboleda G, Archila M.P, Polo F, Segura O. Sobreexpresión de her2 por inmunohistoquímica en cáncer gástrico en el hospital de san José 2005 a 2009.

Fundación Universitaria de Ciencias de la Salud. Cañón B, Hernández J.I, Diaztagle J.J, Galvis D, Galván L. Fiebre de origen desconocido. Un reto clínico. Casos Hospital de San José e Infantil de San José

Fundación Universitaria de Ciencias de la Salud. Archbold G, Gómez M, Enciso C, Rojas M, Taboada H, Segura O. Hipoperfusión en el paciente crítico diagnóstico y seguimiento mediante saturación venosa continua.

Fundación Universitaria de Ciencias de la Salud. Fiorillo A.A, Rodríguez H.A, Monsalve D.J, Segura O. ¿La ligadura de la arteria circunfleja medial profunda disminuye la incidencia de transfusión en reemplazo total de cadera primario?

Fundación Universitaria de Ciencias de la Salud. Lleras J.D, Acero F.A, Pabón M.G, Aldana G.E. Alanino aminotrasferasa en pancreatitis aguda de origen biliar.

Fundación Universitaria de Ciencias de la Salud. Niño C.G. Estudio de costo-efectividad de buprenorfina transdérmico versus oxicodona oral y fentanilo transdérmico en el manejo del dolor crónico no oncológico en el Hospital de San José de Bogotá D.C. enero-diciembre 2010.

Fundación Universitaria de Ciencias de la Salud. Orduz J, Chacón N.P, Rozo G.E, Beltrán D.F, Ospina J.C. Prevalencia de los factores de riesgo cardiometabólico en la población de estudiantes de posgrado y docentes de la Facultad de Medicina de la Fundación Universitaria de Ciencias de la Salud. Segundo semestre 2010.

Fundación Universitaria de Ciencias de la Salud. Orduz J, Pérez L.C, Maecha L, Prieto J, García A, Carvajal D, Corredor D, Pérez D, Camelo J, Portela A, Rivera M.A, Aristizabal M. Prevalencia de los factores cardiometabólicos en el Ministerio de la Protección Social en el marco del proyecto de organizaciones saludables. Bogotá 2011.

Fundación Universitaria de Ciencias de la Salud. Velasco K. Posturas adoptadas por profesionales en instrumentación quirúrgica durante procedimientos quirúrgicos según el método OWAS en una institución de IV nivel de Bogotá en el 2012. Prueba piloto.

Fundación Universitaria San Martín. Muñoz N, Osorio J.D, Pinzón E. Conocimientos y prácticas de las mujeres frente al autoexamen de la mama en una universidad de la ciudad de Cali.

Grupo OXIGENAR. García F, Riveros E. Comparación de variables fisiológicas cardiorespiratorias durante el ejercicio entre estudiantes de medicina sedentarios y acondicionados.

Pontificia Universidad Javeriana. Figueredo M.d.C. Echeverri C.M, Conde A.C, Durango R.M, Flórez D.E, Forero J.C, Fuentes J.A, Gamarra G.D, Cadena Y, Gómez L.F. Bebidas en los ambientes escolares: marketing, publicidad, disponibilidad y precios.

Universidad Cooperativa de Colombia. Caro A, González J.M, Franco S, Mosquera J, Cardona A. Conocimientos sobre primeros auxilios en estu- diantes de grado 10 y 11 de instituciones educativas de Medellín y Bello durante el año 2012.

Universidad de Boyacá. Castañeda A, Pérez I, Bernal T, Suescún S. Prevalencia de parasitosis intestinales y factores de riesgo asociados en escolares y adolescentes del colegio Chicamocha Kennedy I del municipio de Tuta Boyacá.

Universidad de Los Andes, CeiBa, Universidad Jorge Tadeo Lozano. Zambrano J.M, Lemoine P, Ramírez A, Ovalle J, Sarmiento O.L, Zarama R, Cordovez J.M, Pinzón J.D, Pratt M. Actividad física, transporte y salud en Bogotá: el caso de transmilenio.

Universidad de Los Andes, Fundación Santa Fe. Salazar MC, Ramírez A Higuera D, Acosta JC, Leal D, González LV, Ríos AP, Lemus N, Sarmiento OL, Segura E, Vera JF. Actividades sedentarias y prevalencia de sobrepeso y obesidad en niños y adolescentes: el caso de televisión y/o videojuegos.

Universidad de Los Andes, Universidad de Chile, Profamilia, Instituto Nacional de Salud. D. Leal, Ramírez A, Sarmiento O.L, Mahecha M.P, Bravo A, Pinzón P, Samper B, García S, Mosquera T, Atalah E, Ojeda G, Forero Y, Pedraza C, Grijalba C. Estado nutricional durante la gestación en las mujeres colombianas: la coexistencia del bajo peso en adolescentes y el sobrepeso en mujeres adultas.

Universidad de Los Andes. Cleves D, Sarmiento Ol, Ramirez A, Garcia Mp, Campaz D, Vera Jf, Gempeler, Rodriguez M. Análisis multivariado de factores asociados con discordancia en la autopercepción de la imagen corporal en la población adolescente colombiana.

Universidad de los Andes. Donado C, García M.P, Duperly J. Riesgo caF diovascular asociado al ejercicio en corredores aficionados de una carrera recreativa en Bogotá.

Universidad del Magdalena. Suárez Y, Rodríguez U. Inteligencia emocional e ideación suicida en estudiantes de psicología de una universidad pública del caribe colombiano.

Universidad Industrial de Santander. Guarín S.K, Chaves M, Arenas L.K, Pinilla N, Rueda O.L. Variables predictoras del desempeño del médico general colombiano en el primer examen nacional de conocimientos en electrocardiografía.

Universidad Industrial de Santander. Meza L, Calvo L, Muñoz D. R, Vargas C.López E, Delgado M, Morillo C.A, Rueda O.L. Variabilidad de la frecuencia cardíaca (VFC) como factor pronóstico de mortalidad en pacientes con infarto del miocardio: revisión sistemática de estudios observacionales - Meta - Análisis

Universidad Militar Nueva Granada. Holguin D.F, Méndez I.A, Pachón D.P, Africano J.F, González I.M, Rojas N.A. Susceptibilidad antimicrobiana de cepas de Staphylococcus aureus aisladas en individuos sanos.

Universidad Militar Nueva Granada. Rodríguez J, Prieto S, Correa C, Arnold Y, Alvarez L, Bernal P, Mora J, Soracipa Y, Rojas N, Pineda D. Dinámica de la epidemia del dengue en Colombia. Predicciones de la trayectoria de la epidemia.

Universidad Simón Bolívar-Barranquilla. Behaine A, Brunal S, Acosta C, Figueroa G, De La Torre W. Estado nutricional de los niños de 1 a 12 años que asisten a comedores de los C.A.M.I.N.O (sur occidente, Las Américas, Ciudadela Metropolitana) de la ciudad de Barranquilla en el segundo período del año 2011

Universidad Simón Bolivar-Barranquilla. Macias K, Beleño E, Baquero W, Rueda M, Ospino W, Rubio L.D. Prevalencia del consumo de sustancias psicoactivas en la población escolarizada de 10-20 años de edad, en el distrito de Barranquilla en el año 2011.

Universidad Tecnológica de Pereira. Buitrago J, Sánchez J.C, Guerra Alvaro, García S.V, Lucumi L.J, Romero C.R. Evaluación del cambio de actitud después de una intervención pedagógica lúdica sobre donación y trasplante de órganos en la comunidad de Risaralda-Colombia.

Universidad Tecnológica de Pereira. Echeverri L.F, Londoño M.J, Ochoa S.A, Romero C.R, Ruiz J.O. Impacto de un programa de terapia del humor en la unidad pediátrica del Hospital San Jorge de Pereira. 